BNL--31955

DE83 002836

$\operatorname{lin}_{0 \mathrm{Oc} 635}^{31955}$

SEARCH FOR MASSIVE PHOTON PAIR PRODUCTION

AT THE CERN INTERSECTING STORAGE RINGS

C. Kourkoumelis and L.K. Resvanis

UNIVERSITY OF ATHENS, GREECE

T.A. Filippas and E. Fokitis

NATIONAL TECHNICAL UNIVERSITY, ATHENS, GREECE

R.B. Palmer, D.C. Rahm, P. Rehak and I. Stumer BROOKHAVEN NATIONAL LABORATORY ${ }^{1}$, UPTON, NEW YORK

C.W. Fabjan, T. Fields ${ }^{2}$, D. Lissauer ${ }^{3}$, I. Mannelli ${ }^{4}$, P. Mouzourakis ${ }^{5}$, A. NapPi ${ }^{4}$ and W.J. W111is

CERN, GENEVA, SWITZERLAND

M. GoIdberg
SYRACUSE UNIVERSITY
, SYRACUSE, NY

${ }^{1}$ This research was supported by the US Dept. of Energy under Contract No. DE-AC02-76CH00016.

${ }^{2}$ permanent address: Argonne National Laboratory, Argonne, IL

'Permanent address: Tel-Aviv University, Israel

${ }^{4}$ On leave of absence from the University of Pisa and the INFN, Sezione di Pisa, Italy

5 Present address: CEN-Saclay, Gif-sur-Yvette, France

6 Work supported by the US National Science Foundation

The subritted manuscript has been authored under contract DE-AC02-76CE00016 with the U.S. Department of Energy. Accordingly, the U.S. Government retains a nonexclusive, royalty-free license to publish or reproduce the published form of this contribution, or allow others to do so, for U.S. Governinent purposes.

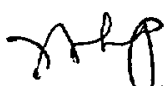

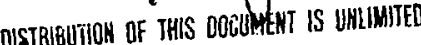




\title{
SEARCH FOR MASSIVE PHOTON PAIR PRODUCTION AT THE CERN INTERSECTING STORAGE RINGS
}

\author{
C. Kourkoumelis and L.K. Restanis \\ University of Athens. Greece \\ T.A. Filippas and E. Fokitis \\ National Technical University, Athens, Greece \\ R.B. Palmer, D.C. Rahm, P. Rehak and I. Stumer \\ Brookhaven National Laboratory", Upton. NY, USA

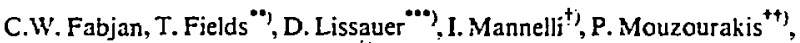 \\ A. Nappi ${ }^{\dagger}$ and WJ. Willis \\ CERN, Geneva. Switzerland \\ M. Goldberg \\ Syracuse University ${ }^{+++)}$, Syracuse. NY, USA
}

\begin{abstract}
A search for massive pinoton pair production at $\sqrt{ } s=63 \mathrm{GeV}$ has been carried out on the data sample previously employed for the electron pair production study. Positive evidence is reported for $m_{w}>6 \mathrm{GeV}$, with a production cross-section similar to Drell-Yan electron pairs. The ratio $\gamma / \pi^{0} \pi^{0}$ was measured to be $\sim 10^{-3}$ for a $p_{\tau}$ of each $y$ or $\pi^{0}$ above $3 \mathrm{GeV} / \mathrm{c}$.
\end{abstract}

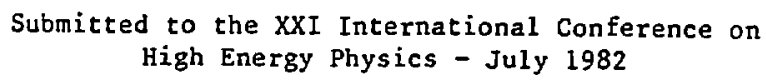

*) This research was supponed by the US Department of Energy. under Contract No. DE-AC02-76CH00016.

**) Permanent address: Argonne National Laboratory, Argonne, Ill., USA.

**) Permanent address: Tel-Aviv University, Israel.

t) On leave of absence from the University of Pisa and the INFN, Sezione di Pisa, Italy.

+†) Present address: CEN-Saclay, Gif-sur-Yvette, France.

$+++)$ Work supported by the US National Science Foundation. 


\section{INTRODUCTION}

Direct two-photon production in hadronic interactions has been discussed in the theorctical literaturc by many authors $[1-4]$.

We present here the first experimental evidence for the process $p p \rightarrow z y+X$ at is $=63 \mathrm{GeV}$, when each photon emerges near $90^{\circ}$ in the centre of mass with a transverse momentum of at least $2 \mathrm{GcV} / \mathrm{c}$.

The lowest-order QCD diagrams are shown in Fig. 1. The sub-processes (a) $q \bar{q} \rightarrow \eta n$ and (b) $g \unrhd \rightarrow \eta n$ dominate the cross-section, and at our energies their relative contributions at $90^{\circ}$ have been estinated to be $(\mathrm{gg} \rightarrow \mathrm{h}) t$ $(q \bar{q} \rightarrow 7 n) \sim 0.15$ to $0.30[2]$. Ai the leve] of the parton model. process (a) involves the same structure functions as does the Drell-Yan process $\mathrm{pp} \rightarrow \mathrm{e}^{-} \mathrm{e}^{-}+\mathrm{X}$ (Fig. 1n). At the QCD level, the two-photon production gives additional information. being sensitive to the quark propagator which relates the process to the single direct photon production. The $\mathrm{q} \overline{\mathrm{q}} \rightarrow \bar{r} \boldsymbol{y}$ process is also sensitive to the charge of the quark to the fourth power. Wc have calculated (to first-order $\mathrm{QCD})$ the cross-sections for $\mathrm{pp} \rightarrow \pi ;+\mathrm{X}$ and $\mathrm{pp} \rightarrow d\}+\mathrm{X}[5]$, and these are displayed in Fig. 2a for the $90^{\circ}$ configuration and in Fig. $2 \mathrm{~b}$ for a $20^{\circ}$ back-to-back production. As one can see in Fig. 2a we expect the two photons to he produced with cross-sections similar to those of the dileptons at $90^{\circ}$. A comparison of the two processes for the configurations of $\mathrm{Fig}$. $2 \mathrm{~b}$ could provide a significant test of the quark propagator.

In this paper we will compare the $\%$ cross-section with the $\ell l$ production at $90^{\circ}$. The advantage of normalizing the $\gamma$ data to the Drell-Yan events is that part of the higher-order $O C D$ corrections are the same in both processes [4].

The production environment of the photon and electron pairs is expected to be similar. Photons produced according to the diagrams of Figs. la and $\mathrm{lb}$ are emitted with equal and opposite transverse momentum and unaccompanied by hadrons. In our analy'sis, we have selected $; r$ events which were not accompanied bl hadrons. This requirement will not bias the $\gamma / \ell l$ ratio, but will suppress background due to various mechanisms of bremsstrahlung photon production. described in Figs. 1c. 1d, and le.

\section{THE DATA SAMPLE AND THE METHOD OF ANALYSIS}

In the followine we describe the method of analysis adopted in order to establish experimentally the existence of direct photon pairs in the presence of the mucn more abundant high- $p_{\mathrm{T}}$ production of oppesite-side $\pi^{0}$ pairs.

The sample of data is basically the same as that previously used for the siudy of large-mass electron pair production [6], of $\pi^{0}$ inclusive cross-section at high $p_{\tau}$ 17], and of $\pi^{0} \pi^{0}$ correlations [8]. It corresponds to an integrated luminosity of about $8 \times 10^{37} \mathrm{~cm}^{-2}$. The four detector moduies (Fig. 3) were arranged in two opposite pairs (conventionally labelled "Up" and "Down") around the ISR interaction region. The effective plane for the determination of the coordinates of electromagnetic showers in the lead/liquid-argon calorimeters $u$ as at a distance of $85 \mathrm{~cm}$ from the interaction centre. Each calorimeter covered a solid angle of about $0.9 \mathrm{sr}$ in the laboratory and was segmented laterally and longitudinally to provide measurement of the electron-photon energy and to enhance the hadron rejection.

The events were recorded whenever a localized energy deposition, above a suitable trigger level. was detected in at least one Up and one Down caiorimeter.

A scries of proportional chambers, plastic scintillator hodoscopes, and lithium foil transition radiators, followed by xenon proportional chambers. were fully operational and used in the an alysis. but were not required in the trigger.

The trigger efficiency (Fig. 4a) is essentially the same for electrons and photons.

In the absence of a magnetic field, we consider that with our apparatus the only possible difference between the acceptances of $\because \ddot{y}$ and $\ell l$ pairs could arise from different production kinematics. From Fig. 4b we conclude that there is a difference of at most $15 \%$ bctween the acceptance of Drell-Yan pairs with a decay angular distribution proportional to $1+\cos ^{2} \theta$. and the acceptance of a resonance source decaying isotropically into two photons.

The reconstruction of the electromagnetic showers has been carried out in the same way as for the data on electron pairs [6| and on direct photon production [9] already published. Similarly. the performance of the calorimeters and of the associated sof tware has been simulated with the well-tested EGS [10| Monte Carlo. With the trigger threshold used here, the useful range for the $p_{\tau}$ of a detected $\pi^{\circ}$ or photon starts at about $2 \mathrm{GeV} / \mathrm{c}$ and extends up to $9 \mathrm{GeV} / \mathrm{c}$. The relatively small distance between the calorimeters and the interaction region implies a correspondingly high probability that the photons from a high $p_{\tau} \pi^{0}$ will merge into a singie shower. Ai lower $p_{\tau}$ the dominant source of single photon candidates is the limited geometrical acceptance of the two direct photons. To limit these effects in the data used in the present analysis, we imposed the same selection criteria as were previously used for the direct photon studies [9]. In particular, this applied to the fiducial volume; to the radius and longitudinal development of the shourers; to the absence of additional showers in the calorimeter where the photon or $\pi^{0}$ is detected: and finally, to the absence of any sizeable energy $(100 \mathrm{MeV})$ left unassigned after reconstructing all showers of the event.

In order to search for a signal in the most sensitive and reliable way, the basic quanity we studied is the ratio $R$ of the number of events with a single detected shower to the number of events with a $\pi^{0}$ detected in the same $p_{\tau}$ range, in a given calorimeter. We will use the subscript $s$ or $\pi$ to distinguish the case where the paricle detected in one calorimeter is in coincidence either with a single shower $\left(R_{3}\right)$ or with a $\pi^{0}\left(R_{n}\right)$ detected in one of the opposite calorimeters in the same $P_{\mathbf{T}}$ range. 
The comparison of $R_{5}$ with $R_{T}$ permits us to search for $\because 7$ crents without being sensitive to the precise values of detection efficiencies. The argument is qualitatively as follows: for the case (which is experimentally the dominant one) where $\pi^{\prime \prime} \pi^{\prime \prime}$ pairs are produced. each single shower detected would actually be the consequence of a $\pi^{0}$ misidentification: given the symmetry and the fact that the probability of misidentification of a $\pi^{0}$ in one calorimeter is not correlated with the probability of misidentification in the caiorimeter where the second clement of the pair is derected. it clearly follows that $R_{s}=R_{\pi}$. Since a single photon has no chance of being mistakenly detected as a $\pi^{0}$, the genuinc $\pi^{0}$ events (i.e. a direct photon, whose $p_{\mathrm{T}}$ is balanced by a recoiling jet wirh a $\pi^{0}$ as a leaoing particic), cannot give rise to detected $\pi^{0} \pi^{0}$ events. A. sample of events consisting of a mixture of $\pi^{0} \pi^{6}$ and $; \pi^{0}$ cvents would hence give $R_{s}<R_{T}$ On the contrary. adding true $; z$ events to a $\pi^{0} \pi^{0}$ sample would result in $R_{5}>R_{\tau}$ In conclusion. experimental values of $R_{5} / R_{7}>1$ represent evidence for $\because{ }^{\prime}$ events.

More quantitatively, let us denote by

$N_{\pi \pi}$ the number of $\pi^{0} \pi^{0}$ cients,

$N_{\eta z}$ the number of events with a single $y$ and a recoil $\pi^{0}$.

$N_{w}$ the number of $\because ;$ events.

where the $j^{\prime}$ s and $/ 0 z^{0}{ }^{\circ}$ s are pointing to opposite pairs of calorimeters. each having a $p_{\mathrm{T}}$ above a given threshold.

Let us also define

$P_{t \pi} \rightarrow \pi \pi \quad$ as the probability that a pair of $\pi \pi^{\circ}$ s will be detected as such:

$P_{7+} \rightarrow s \pi$ as the probability that a pair of $\pi^{0 *} \mathrm{~s}$ will be detected as a single shower and $\mathrm{a} \pi^{0}$ :

$P_{a \pi} \rightarrow S S$ as the probability that a pair of $\pi^{n+} s$ will be detected as a pair of single showers;

$P_{r \tau} \rightarrow S \pi \quad$ as the probability that a $j^{0} \pi^{0}$ event will be detected as a single shower and a $\pi^{0}$ :

$P_{p \pi} \rightarrow s s \quad$ as the probability that a $; \pi^{0}$ event will be detected as a pair of single showers;

$P_{r p} \rightarrow s s \quad$ as the probability that a $\because F e v e n t$ will be detected as a pair of single showers.

The number of observed events will then be:

$$
\begin{aligned}
& O_{s s}=N_{\mathrm{v}}\left(P_{\mathrm{Tr}} \rightarrow s s\right)+N_{\mathrm{vT}}\left(P_{v 7} \rightarrow s s\right)+N_{v v}\left(P_{v w} \rightarrow s s\right) \\
& O_{5 \pi}=N_{T \tau}\left(P_{T \pi} \rightarrow 5 \pi\right)+N_{i \tau}\left(P_{j \pi} \rightarrow 5 \pi\right) \\
& O_{79}=N_{47}\left(P_{7 i} \rightarrow \pi \pi\right) \text {. }
\end{aligned}
$$

It should be noted that real $; z$ events coniribure only to $O_{s s}$, and that in the above formulae for $O_{s s}$ and $O_{s q}$ we actually include all neutral meson contributions $(\bar{\pi} . \eta \ldots .$.$) . By definition$

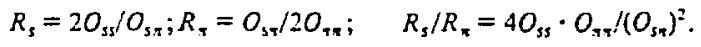

The above detcction probabilitics clearly depend on the geometry of the detectors, on their response to photons. and on the correlations between the two particles of the pair.

Figure $5 \mathrm{a}$ shows, as a function of $\bar{p}_{\mathrm{T}}$, the Monte Carlo calculated probability that a $\pi^{\mathbf{0}}$ with both decay photons incident on a calorimeter is misidentified and accepted as a single shower (open circles) or that it is instead reconstructed as an accepted $\pi^{0}$ (solid dots). Figure 5 b shows the probability that a single photon is accepted as a single shower. The oscillation in the points reflects the statistical accuracy of the Monte Carlo. Clearly, above $6 \mathrm{GeV}$ the probabiiity that the decay photons of a $7^{0}$ merge into a single shower becomes important. In order to calculate the detection probabilities for pairs, needed to predict $R_{5}$ and $R_{v}$, a full Monte Carlo simulation of the apparatus has been carried out inciuding the measured $\pi^{0} \pi^{0}$ correlations $[8]$ and the contribution, according to the measured cross-sections [11\}, of other neutral mesons such as $\eta, \omega$, and $\eta^{r}$. which produce high- $p_{T}$ photons in their decays. As far as $; \pi^{0}$ events are concerned. from an independent study of the correlations $|12|$ between a $\pi^{0}$ or a high- $p_{T}$ single direct photon and the charged particles in the recoil jet. we have checked that they are correlared in the same way as $\pi^{\circ} \pi^{0}$ pairs: in place of the $\left(N_{r \tau} / N_{T \pi}\right)$ ratio we have therefore used as input for the Monte Carlo the measured ratio $; / \pi^{0}\{9\}$ of the respective single-particle inclusive cross-sections.

Figures 6,7 , and 8 give the results of the calculation for $R_{s}$ and $R_{n}$ for various trigger threshold values of the $\pi^{0}$ or shower of lowest $p_{T}$ and as a function of the $p_{\mathrm{T}}$ of the other partner of the pair. The contributions of the different sources to $R_{5}$ and $R_{4}$ are shown separately. It should be remarked that for pairs of mesons the Monte Carlo, including the correlation cffects, does indecd give $R_{5}=R_{n}$. At relatively low $p_{\mathrm{T}}$. the most important contribution comes from $\pi^{0} \bar{\pi}^{0}$ and $\pi^{0}$-neutral meson pairs. At ingher $p_{T}$ the dominant source is given by $\pi^{0}$ events and this differentiates slightly between $R_{s}$ and $R_{\pi}$, the $R_{s}$ remaining alw'ays smaller than $R_{n}$.

Figure 9 summarizes the expected dependence of the ratio $R / R_{n}$ in the absence of a real $j r$ signal. Figure $10 \mathrm{a}$ shows the values measured experimentally for $R_{s}$ (solid dots) and $R_{n}$ (open circles), plotted with their statistical error bars. The Monte Cario calculated values are also shown for comparison $\left(R_{7}: \ldots, \ldots, R_{s}: \ldots\right)$. The experimental values for $R_{3}$ are always higier than for $R_{7}$, contrary to expectation and the Montc Cario prediction in the absence of a real $\because \%$ signal. Instead, the values of $R_{n}$ agree well with the corresponding Monte Carlo calculations. 
The measured value of $R_{\mathrm{s}} / R_{\mathrm{r}}$ and the same ratio calculated in the absence of a real $\eta$ source are displayed in Fig. $10 b$ for the three different $p_{\mathrm{T}}$ thresholds.

Because each $\pi^{0}$ carries a different fraction of the momentum of its jet. we expect the real $;$ events to manifest a better balance of their transverse momentum than the background originating in $\pi^{0} a^{0}$ pairs. The quantity $z=p_{T_{1}} X$ $\cos \theta / p_{\mathrm{T}_{2}}$ describes the transverse momentum balance of a pair. In Fig. 11 we display the $R_{s} / R_{\text {, }}$ ratio for balanced pairs $(z \approx 1)$ and for unbalanced pairs $(z \neq 1)$ for $p_{T}$ trigger $>2 \mathrm{GeV} / \mathrm{c}$. The background will contribute to the unbalanced pairs, and their $R_{s} / R_{n}$ ratio (open circles) is indeed lower than the same ratio for balanced pairs (open triangles); it is in good agreement with our Monte Carlo prediction (solid line).

The difference between the measured value of $R_{\mathrm{s}} / R_{\mathrm{T}}$ and the value calculated for it on the assumption that no real $\eta$ source exists is directly related, according to the definitions given in (1) and (2). to the number $\left(O_{s s}\right)_{k r}$ of real $\because ;$ events contained in the observed sample.

$$
O_{s s} i_{\pi i}=\frac{1 O_{s \pi} i^{2}}{4 O_{z \pi}}\left\{\left(\frac{R_{s}}{R_{s}}\right)_{\text {measurce }}-\left(\frac{R_{s}}{R_{s}}\right)_{\mathrm{sc} \text { for no } \pi i}\right\}
$$

\section{RESULTS}

Table 1 summarizes the results obtained. In order to ensure the greatest freedom from systematic crrors. the above analysis was performed using only the calorimeter information. As a consequence, the observed net $n$ signal $\left(O_{s s}\right)_{y z}$ still includes, in addition to any detected true $\because ;$ event. electron pair events which up to this stage of the analysis are accepted with essentially the same efficiency, and which display the same correlation. The electron pairs can, however, be easily distinguished and removed by using the additional information on charged tracks provided by the scincillation hodoscopes, the cylindrical multiwire proportional chambers (MWPCs), and the linear MWPC coupled to the lithium foil transition radiators which precede the liquid-argon calorimeter. From the previous study of electron pair production $[6]$ we know that in $(87 \pm 5 \% \%$ of cases an electron produces a recognized track in these detectors. In order to make sure that we subtract the electron pairs. we have accepted as such all cvents in which both detected singic showers are accompanied by a charged track within a $10^{\circ}$ cone around the shower, without imposing any other requirement. We have subsequently checked that the events sejected in this way are indeed electron pairs. because in fact $80 \%$ of them satisfy the full set of criteria imposed during the original analysis for electron pairs. In addition. their mass spectra. shown in Fiy. 12. are quite compatibie with the expected ones. given the $p_{T}$ cut on each shower. and they clearly show the $Y$ resonance. This confirms the validity and the sensitivity of the method of analysis. The number of events (see Tabie 1) obtained by direct subtraction, after correction for track-finding incficiency but without attempting to remove possible background in the electron pair sample, is hence a safe. lower limit of the true number of $\dddot{i}$ events [13]. For example, for $\mu_{\mathrm{T}}>3 \mathrm{GeV} / \mathrm{c}$ we observe $119 ; ;$ candidates. The uncorrelated background is estimated to be $63 \pm 10$ events, assuming a ratio $R_{s} / R_{\mathrm{g}}=0.89$ which was predicted by our Mfonte Cario. By subtraction we obtain a combined signal of $(56 \pm 15) i r+e^{+} e^{-}$events.

Repeating the whole procedure for showers and $\pi^{\circ}$ which do have associated tracks in a $10^{\circ}$ cone around them, we obtain an $e^{-} e^{-}$signal c: $19 \pm 4.5$ events. This is an overestimate of the real dilepton signal because it will contain $; z$ events which happen to overlap with tracks in the $10^{\circ}$ cone. After correction for track inefficiency, we estimate our dilcpton signal in this sample to be $25 \pm 6$ events. By subtraction, the net $\%$ signal is $31 \pm 16$ events. In summary. for $p_{\mathrm{T}}>2 \mathrm{GeV} / \mathrm{c}$ no statistically significant net $\%$ signal is obtained becalise of the size of the background; for both $p_{\mathrm{T}}>$ $3 \mathrm{GeV} / \mathrm{c}$ and $p_{\mathrm{T}}>4 \mathrm{GeV} / \mathrm{c}$ we are left with a number of $\%$ events that is more than three times the uncertainty in the number of events expected in absence of a true $; /$ signal.

Since in the liquid-argon calorimeters the detection efficiency of a high-energy photon or electron is essentially the same, we can directly determine the ratio of $\%$ to electron pair events, within the geometrical acceptance of the apparatus and the imposed $p_{\mathrm{r}}$ cuts. In order to derive the ratio of the $\boldsymbol{\gamma} /$ cross-sections to the Drell-Yan electron pair continuum production (which is interesting for the theoretical comparison, as mentioned in the Introduction), we have subtracted the contribution from the $Y^{\prime}$ resonances and assumed that the production distribution for $\eta^{\prime}$ and the $\mathrm{e}^{+} \mathrm{e}^{-}$ continuum are the same.

Table 2 summarizes the relevant quantities for the calculation of this ratio. We estimate that the ratio $r / \mathrm{c}^{+} \mathrm{e}^{-}$ continuum $=1.7 \pm 1$ for $p_{T}>3 \mathrm{GeV} / \mathrm{c}$. and $d^{2} \sigma / \mathrm{d} m \mathrm{nd} y \cdot \mathrm{v}_{\mathrm{n}}=(8 \pm 4) \times 10^{-35} \mathrm{~cm}^{2} / \mathrm{GeV}$ for $8 \leq m_{r F} \leq 11 \mathrm{GeV}[13]$.

It is also interesting to compare the $\pi$ signal with the $\pi^{0} \pi^{0}$ production for the same kinematic $p_{T}$ range. In particular, this makes it possible to evaluate the size of the possible spurious sources that could contribute to the $r 7$ signal detected by us. The number of observed $\pi^{0} \pi^{0}$ events is. 185 for $p_{T}>3 \mathrm{GeV} / \mathrm{c}$ and 22 for $p_{T}>4 \mathrm{GeV} / \mathrm{c}$. The corresponding net $\eta$ signals observed are 31 and 7 . The Monte Carlo program gives a ratio of $1.2 \times 10^{-2}$ for the relative acceptance of a $\pi^{0} \pi^{0}$ pair over that for a $\gamma$ pair for the same kinematics and $p_{\mathrm{r}}$ range. The difference between the $\pi^{0} \pi^{\circ}$ and $\eta$ acceptance comes largely from the localized energy deposition required in the trigger. Because additional charged hadrons are found experimer: $\bullet ! y[11]$ to be more frequently associated with $\pi^{0 *} s$ rather than with direct 
photons, we expect that ther stringent requirement on the unassigned energy may further depress the relative $\pi^{0}$ acceptance. We have indeed found that without the unassigned energy requirement. the number of accepted $\pi^{0}$ pairs increases by a factor of 1.7. Taking this effect into account, we finally estimate

$$
\% / \pi \frac{0}{10}=(1.2 \pm 0.6) \times 10^{-3} \text { for } p_{\mathrm{T}}>3 \mathrm{GeV} / \mathrm{c}
$$

and

$$
\because / \pi^{0} \pi^{0}=(2.6 \pm 1.8) \times 10^{-3} \text { for } p_{\mathrm{T}}>4 \mathrm{GeV} / \mathrm{c}
$$

\section{BACKGROUNDS}

Uncorrelated pairs of mesons $\left(\hat{\pi}^{0}, \eta, \omega, \eta^{\prime}\right)$ decaying into photons were estimated and subtracted (see Table l).

We have considered the following additional sources of hackground .2 the signal:

- Dileptons ceuld simulate diphotons if the track-finding algorithm was inefficient for both tracks. This inefficiency was estimated to be $(2 \pm 1) \%$ causing an enhancement of the $\%$ signal by $0.5 \pm 0.3$ events for $p_{T}>3 \mathrm{GeV} / \mathrm{c}$ and $0.3 \pm 0.2$ events for $p_{\mathrm{T}}>4 \mathrm{GeV} / \mathrm{c}$.

- We have studied in detail the possible size of a $\eta^{\prime \prime}$ signal due to anomalously high production of $\eta \eta$ or $\eta^{\prime} \eta^{\prime}$ pairs. although there is no experimental [8] or theoretical indicaticn of the presence of such pairs. We estimate that a complete correlation of $\eta^{\prime}$ s and $\eta^{\prime \prime}$ s (i.e. if cvery $\eta$ or $\eta^{\prime}$ produced at $90^{\circ}$ would always be accompanied by an $\eta$ or $\eta^{\prime}$ in the opposite side) could be responsible for $\sim 20 \%$ of the observed $\gamma$ signal.

- Hadrons interacting in the first layers of a calorimeter can simulate a single shower. The rejections by our culorimeters at the trigger level and the tight selection criteria used in our analysis will result in only $0.4 \%$ of charged pions being accepted as a single shower in our $p_{\mathrm{T}}$ range [9]. In order to simulate two-photon events, this will have to hapnen for both showers. After accounting for the different species of hadrons and using our estimate of $i \eta^{1} / \pi^{0} \pi^{0} \sim$ $10^{-3}$ and our track reconstruction efficiency of $0.87 \pm 0.05$, we concluded that the hadronic contamination to the $r i$ signal is negligible.

\section{CONCLUSIONS}

We have presented the first evidence for the process $\mathrm{pp} \rightarrow \%+\mathrm{X}$ at $\mathrm{\gamma}^{\prime} \mathrm{s}=63 \mathrm{GeV}$. The analysis was carried out on a data sample which contains dielectron and diphoton candidates. In order to achieve a strong rejection of the backgrounds. we have used the same selection criteria as that used in our direct photon studies. The method of long-range high- $p_{T}$ correlation between the two single photon or electron candidates proved to have sufficient sensitivity to unravel the $Y$ signal in our sample, just by requiring loose tracks in association with our photon candidates. We report a cross-section

$$
\mathrm{d}^{2} \sigma / \mathrm{d} m \mathrm{~d} \mathrm{l} \psi_{y=0}=(8 \pm 4) \times 10^{-3 \mathrm{~s}} \mathrm{~cm}^{-2} / \mathrm{GeV}
$$

for $\delta \leq m_{r y} \leq \| 1 \mathrm{GeV}$ and a ratio $m / \mathrm{e}^{+} \mathrm{e}^{-}=1.7 \pm 1$ for $p_{\mathrm{T}}(\gamma, \mathrm{e})>3 \mathrm{GeV} / \mathrm{c}$, which is in good agreement with the theoretical expectation.

Our $\because y$ events could also be interpreted as heary $C=+$ states $\left(0^{-+}, 0^{++}, 2^{++}\right)$of the $Y$ family decaying electromagnetically. Lising a brancining ratio of $2 \times 10^{-4}$ for $\eta_{\mathrm{b}} \rightarrow \eta y$ and the cross-section [14]

$$
d \sigma /\left.d y\left(p p \rightarrow \eta_{b}+x\right)\right|_{s=0} \approx 3 \times 10^{-33} \mathrm{~cm}^{-2}
$$

we estimate

$$
\frac{\sigma\left(p p \rightarrow \eta_{b}+X\right) \times b\left(\eta_{b} \rightarrow \eta_{i}^{\prime}\right)}{\sigma(p p \rightarrow Y+X) \times b\left(Y \rightarrow \mathrm{e}^{+} e^{-}\right)}=1 / 20 .
$$

This ratio together with the number of $Y$ events (see Table 2) predicts that at most one event of our $\gamma$ 'sample could be explained by an $\eta_{\mathrm{h}}$ decay.

A comparison of the process $\mathrm{pp} \rightarrow 7 \eta+\mathrm{X}$ with the two-jet production in $\eta$ collisions $[15]$ and with the possibility of massive glueball states [16] materieizizing as a pair of photons could provide additional inderstanding of QCD.

We would like to thank F. Paige, A.P. Contogouris and E. Paschos for useful discussions. 


\section{REFERENCES}

1. S.M. Berman et al.. Phys. Rev. D4, 3388 ( 971$)$.

K. Soh et ai., Phys. Rev. D 18. 751 (1978).

M. Krawegyk and W. Ochs, Phys. Lett. 79B. 119 (1978).

S. Hemmi. Prog. Theor. Phys. 63, 1073 (1980).

2. B.L. Combridge, CALTECH preprint CALT-68-766(1980).

C. Carimalo et al., Phys. Lett 98B, 105 (1981).

3. F. Paige and I. Stumer, Proc. ISA.BELLE Summer Study, Brookhaven. 1981 (BNL-51443. Brookhaven Nat. Lab.. Upton. Ll. New York, 1981).p. 479.

4. A.P. Contogouris et al., Two-photon production at large $p_{\mathrm{T}}$ as a new test of QCD, McGill University preprint (1981).

5. For the calculation of the cross-section $\mathrm{pp} \rightarrow \ddot{z}+\mathrm{X}$, we have used

$$
\frac{\mathrm{d} \sigma}{\dot{\mathrm{a}}}(\mathrm{q} \hat{\mathrm{q}}+\dddot{i})=\frac{2 \pi}{3} \mathrm{a}^{2} \frac{1}{\hat{s}^{2}}\left(\frac{\hat{i}}{\hat{i}}+\frac{\hat{u}}{i}\right)
$$

and the distribution functions of J.F. Owens and E. Reya. Phys. Rev. D 17. 303 (1978).

6. J.H. Cobb et al., Phys. Lett. 72B, 273 (1977).

C. Kourkoumelis et al.. Phys. Lett. 91B. 475 (1980).

7. C. Kourkoumelis et al.. Phys. Lett. 84B. 271 (1979).

8. J.H. Cobb et al., Phys. Rev. Lett. 40. 1420(1978).

C. Kourkoumelis et al., Phys. Lett. 85B, 147 (1979).

C. Kourkoumelis et al.. Nucl. Phys. B158, 39 (1979).

9. C. Kourkoumelis et al.. Phys. Lett. 87B, 292 (1979).

M. Diakonou et al., Phys. Lett. 91B. 296 (1980).

E. Anassontzis et al., High-p direct photon production in pp collisions. CERN-EP/82-26, to be published in Zeitschrift für Physik.

10. R.L. Ford and W.R. Nelson. The EGS code sy'stem: Computer program for the Monte Carlo sinulation of cicctromagnetic cascade showers. SLAC-210(1978).

11. For our $p_{\mathrm{T}}$ range we have measured the following ratios:

$\eta / \pi^{0}=0.55 \pm 0.07, \omega / \pi^{0}=0.87 \pm 0.17, \eta^{1} / \pi^{0}=0.90 \pm 0.25$.

C. Kourkoumelis et al., Phys. Lett. 84B, 277 (1979).

M. Diakonou et al., Phys. Lett. 89B, 43\% (1980).

In the Monte Carjo program these values were assumed to be indeper dent of $p_{T}$.

12. M. Diakonou et al., Phys. Lett. $91 \mathrm{~B}, 301$ (1980).

C. Kourkoumelis et al., Nucl. Phys. B 179, 1 (1981).

13. The ratio and the cross-sections were nct corrected for the fact that real $\because y$ events overlapping with tracks were interpreted as dileptons. We have studied the probability of chance overlap of a isack with a $/$ within our $10^{\circ}$ cone. We concluded that owing to our procedure. $4.7(2.4)$ ir' events could have been lost for $p_{7}>3 \mathrm{GeV} / \mathrm{c}(4 \mathrm{GeV} / \mathrm{c})$.

14. G.J. Aubrecht et al.. Ohio State University preprint COO-1545-250(1980).

R. Baier and R. Rückl. Max-Plank lnst., Munich, preprint MPI-PAE. PTH 16/82(1982).

15. M.Spitzer, DESY Report 80/43(1980).

16. M. Goldberg, Northeastern University preprint NUB 2425 (1980). 
Table 1

Statistics of the observed events

\begin{tabular}{|c|c|c|c|c|}
\hline Momentum & & $p_{\mathrm{T}}>2 \mathrm{GeV} / \mathrm{c}$ & $p_{\mathrm{T}}>3 \mathrm{GeV} / \mathrm{c}$ & $p_{\mathrm{T}}>4 \mathrm{GeV} / \mathrm{c}$ \\
\hline $\begin{array}{l}\text { Observed events } \\
R=R_{s} / R_{7} \text { observed }\end{array}$ & $\begin{array}{l}s s \\
s \pi \\
\pi \pi\end{array}$ & $\begin{array}{c}1773 \\
2643 \\
1097 \\
1.11 \pm 0.05\end{array}$ & $\begin{array}{c}119 \\
229 \\
185 \\
1.68 \pm 0.21\end{array}$ & $\begin{array}{c}27 \\
21 \\
22 \\
5.39 \pm 2.06\end{array}$ \\
\hline$R=R_{s} / R_{\pi}$ Monie Carlo & & $0.99 \pm 0.05$ & $0.89 \pm 0.05$ & $0.76 \pm 0.05$ \\
\hline Calculated background & ss & $1576 \pm 78$ & $63.0 \pm 10$ & $4 \pm 2$ \\
\hline Signal $\ddot{z}+\mathrm{e}^{-c^{-}}$ & & $197 \pm 89$ & $56 \pm 15$ & $23 \pm 5.5$ \\
\hline $\begin{array}{l}\text { Observed events with an associated } \\
\text { track in a } 10^{\circ} \text { cone around } \\
\text { each shower or } \pi\end{array}$ & $\begin{array}{l}s s \\
s \pi \\
\pi \pi\end{array}$ & $\begin{array}{r}110 \\
117 \\
68\end{array}$ & $\begin{array}{r}20 \\
7 \\
13\end{array}$ & $\begin{array}{r}12 \\
1 \\
2\end{array}$ \\
\hline Calculated background to $\mathrm{e}^{-} \mathrm{e}^{-}$events & & $50 \pm 11$ & $0.2 \pm 0.7$ & $0.1 \pm 0.2$ \\
\hline$e^{-} e^{-}$signal & & $60 \pm 15$ & $19 \pm 4.5$ & $12 \pm 3.5$ \\
\hline $\mathrm{e}^{+} \mathrm{e}^{-}$corrected for track inefficiency & & $79 \pm 20$ & $25 \pm 6$ & $16 \pm 4.5$ \\
\hline$y^{\prime}$ (by subtraction) & & $118 \pm 91$ & $31 \pm 16$ & $7 \pm 7$ \\
\hline
\end{tabular}

Table 2

Estimate of the $\gamma ;$ cross-sections

\begin{tabular}{ll}
\hline$p_{\mathrm{T}}$ requirement for each shower & $p_{\mathrm{T}}>3 \mathrm{GeV}$ \\
$\mathrm{e}^{+} \mathrm{e}^{-}$events & $25 \pm 6$ \\
$\mathrm{e}^{+} \mathrm{e}^{-}$events: $8 \leq m_{\mathrm{e}+\mathrm{e}} \leq 11 \mathrm{GeV}$ & $15 \pm 5$ \\
$\left(Y+Y^{\prime}+Y^{\prime \prime}\right) / \mathrm{e}^{+} \mathrm{e}^{-}$continuum: $\left.8 \leq m_{\mathrm{r}-\mathrm{e}} \leq 11 \mathrm{GeV}\right)$ & \\
as measured with same data [6] & $0.9 \pm 0.3$ \\
$Y+Y^{\prime}+Y^{\prime \prime}$ events & $7 \pm 4$ \\
$r / \mathrm{e}^{+} \mathrm{e}^{-}$continuum $=31 \pm 16 / 18 \pm 6$ & $1.7 \pm 1$ \\
$r$ & $(8 \pm 4) \times 10^{-35} \mathrm{~cm}^{2} / \mathrm{GeV}$ \\
\hline
\end{tabular}


Figure captions

Fig. 1: $\%$ production diagrams:

a) $q \bar{q} \rightarrow \because \xi$

b) $g g \rightarrow \%$

c), d), e) various bremsstrahiung photon diagrams simulating $\eta$ ' production

1) Drell-Yan: $q \bar{q} \rightarrow e^{-} e^{-}$

Fig. 2: Comparison of the production cross-sections of diphotons and dileptons in pp interactions at $s=$ $62 \mathrm{GeV}[5]$ :

a) as a function of $p_{\mathrm{T}}$ when both $\gamma^{\prime}$ s and electrons emerge at $90^{\circ}$ :

b) as a function of the ccntre-of-mass angle of one $\gamma$ or electron. when the seconid onc is emitted at $20^{\circ}$ with $p_{\mathrm{T}}=3 \mathrm{GeV} / \mathrm{c}$.

Fig. 3: Geometrical arrangement of the apparatus for the dilcpton and diphoton studies.

Fig. 4: a) Trigger efficiency for single showers

b) Mass acceptance for the geometrical configuration of Fig. 3 for $r$ and $f l$. We have assumed a production that is uniform in rapidity with a $p_{\mathrm{T}}$ dependence $\mathrm{d} . \mathrm{V}^{\prime} / \mathrm{d} p_{\overline{\mathrm{T}}}^{2} \sim \mathrm{e}^{-\delta p_{\mathrm{T}}} . b=1.4 \mathrm{GeV}^{-1}$. The $\mathrm{e}^{*} \mathrm{e}^{-}$ decay distribution follows $\mathrm{d} n / \mathrm{d} \cos \theta=1+\cos ^{2} 9$, when $\theta$ is measured in the 5 -channel helicity frame. We have determined [6] from this dilepton sample: $\mathrm{B}=1.40 \pm 0.15 \mathrm{GeV}^{-1}$ and $\mathrm{d} n / \mathrm{d} \cos \theta=1+$ $(1.15 \pm 0.34) \times \cos ^{2} \theta$. For the acceptance of the $; i$ pairs, we have assumed a resonance with an isotropic decay distribution.

Fig. 5: Monte Carlo calculated probability (including detection and reconstruction efficiency) for:

a) a $\pi^{0}$, with both decay' photons incident on a calorimeter, to be accepted as a singie shower (open circles) or $3 \pi^{\circ}$ (solid dots):

b) a single photon to be identified as a single shower.

Fig. 6: The $1 / \pi^{0}$ ratio calculated by the Monte Carlo program for a $\pi^{0}$ or shower trigger threshold of $p_{T}>2 \mathrm{GeV} / \mathrm{c}$ as a function of the $p_{T}$ of the other member of the pair. $R_{3}$ and $R_{T}$ (see text) are similar in the absence of direct photon source. Note that $R_{\mathrm{s}}$ is below $R_{\tau}$ in the presence of direct photons.

Fig. 7: As Fig. 6 for $p_{\mathrm{T}}>3 \mathrm{GeV} / \mathrm{c}$.

Fig. 8: As Fig. 6 for $p_{\mathrm{T}}>4 \mathrm{GeV} / \mathrm{c}$.

Fig. 9: Monte Carlo calculated dependence of the ratio $R_{s} / R_{\tau}$ on $p_{T}$ for three different threshoids.

Fig. 10: a) The observed $; / \pi^{0}$ ratio when a single shower $\left(R_{s}\right.$, solid dotsi or a $\pi^{0}\left(R_{\mathrm{r}}\right.$. opcn circles) triggered in the opposite side. The Monte Carlo caiculated vaiues are superimposed for comparison: $R_{7} \ldots \ldots . . . R_{s} \ldots . .$.

b) The observed and the Monte Carlo calculated $R_{\mathrm{s}} / R_{\mathbf{z}}$ ratio for three different $p_{\mathrm{T}}$ thresholds.

Fig. 11: The observed $R_{\mathrm{s}} / R_{\tau}$ ratio for $p_{\mathrm{T}}$ "balanced" pairs (open triangies) and for $p_{\tau}$ "unbalanced" pairs (open circles) for the threshold $p_{\mathrm{T}}>2 \mathrm{GeV} / \mathrm{c}$. The solid line represents the Monte Carlo background prediction for $R_{s}, R_{\pi}$.

Fig. 12: Invariant mass spectrum for $\mathrm{e}^{+} \mathrm{e}^{-}$pairs. The $\mathrm{e}^{+} \mathrm{e}^{-}$pair is defined as a $\because z$ pair when each shower is accompanied by a charged track within a $10^{\circ}$ cone. The spectrum was not corrected for geometrical acceptance or trigger efficiency. For the shower reconstruction efiiciency, we have required at most $200 \mathrm{McV}$ of unassigned energy' per caiorimeter (or $100 \mathrm{MeV}$ for the events in the hatched histogram). 


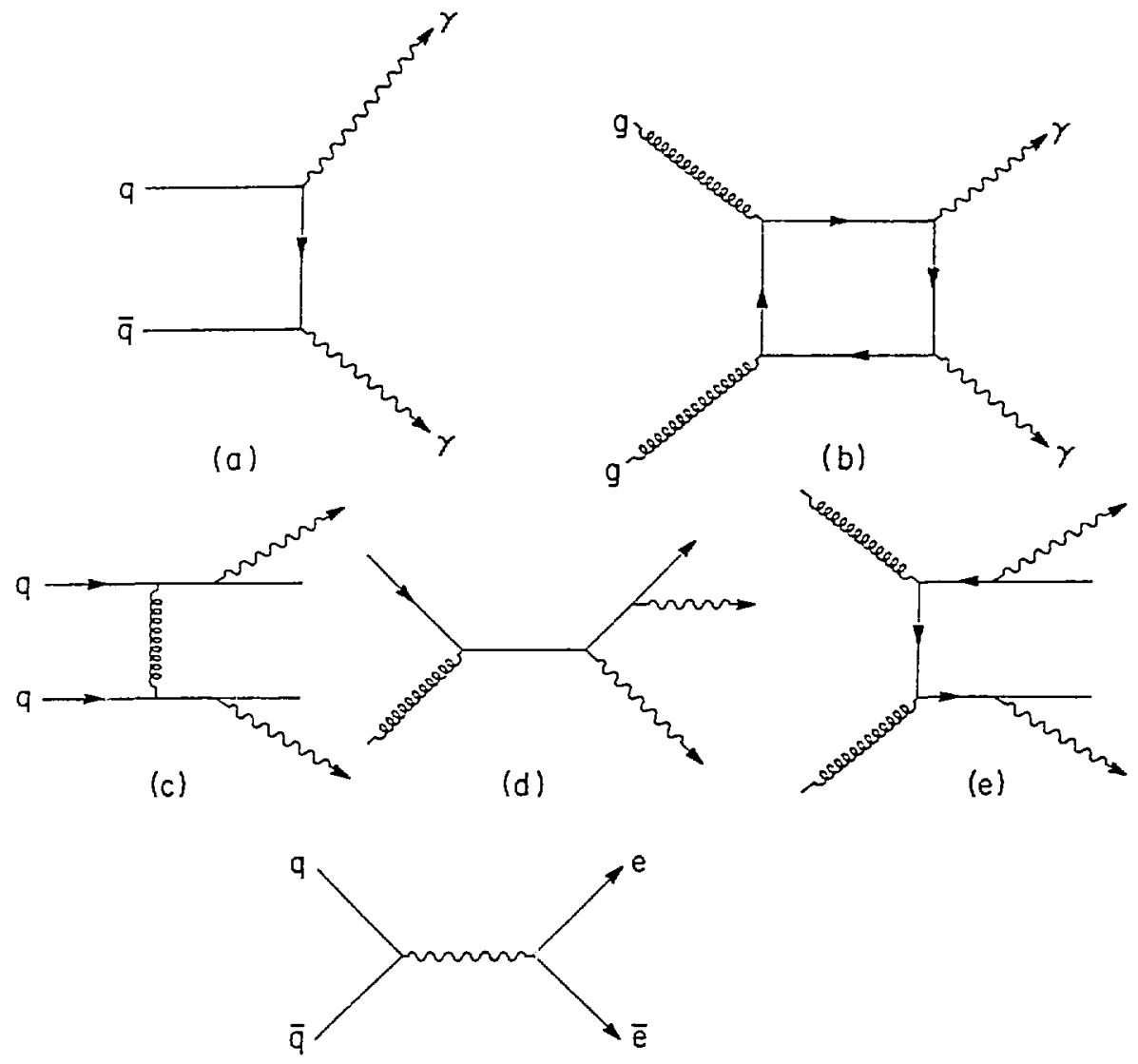

(f)

Fig. I 

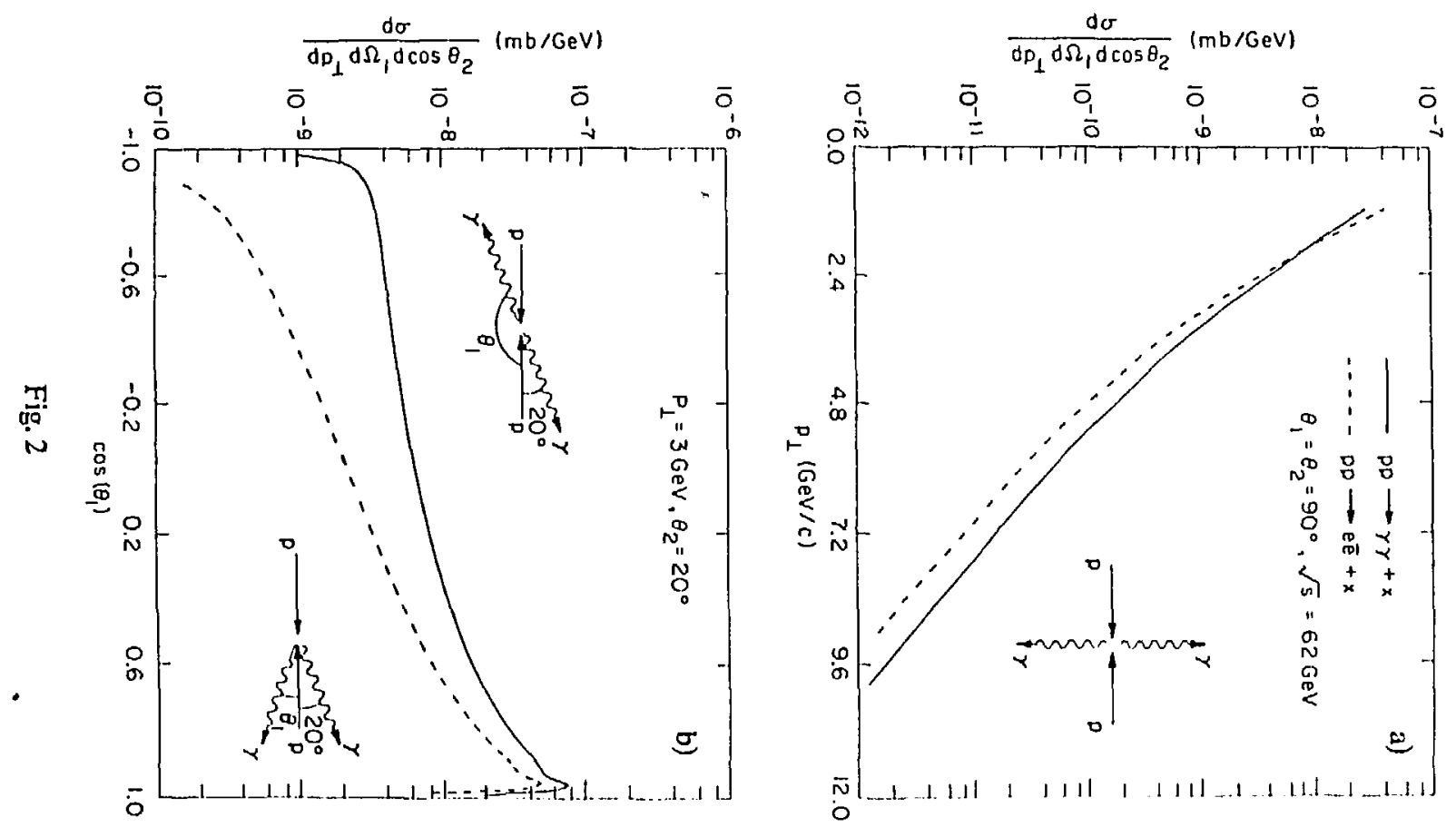


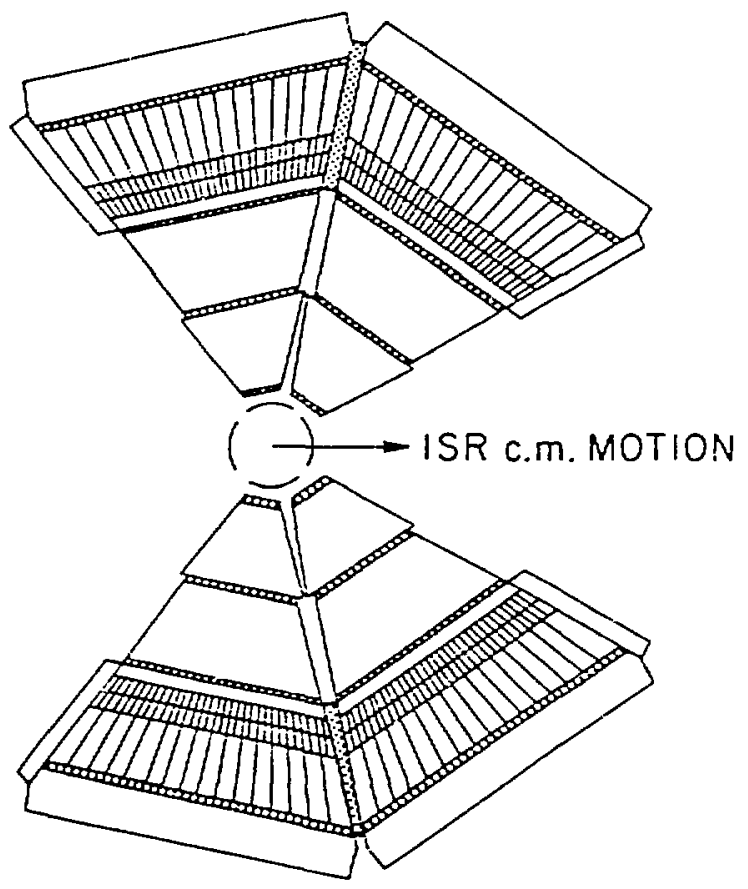

Fig. 3 
GEOMETRICAL ACCEPTANCE B TRIGGER EFFICIENCY (\%)

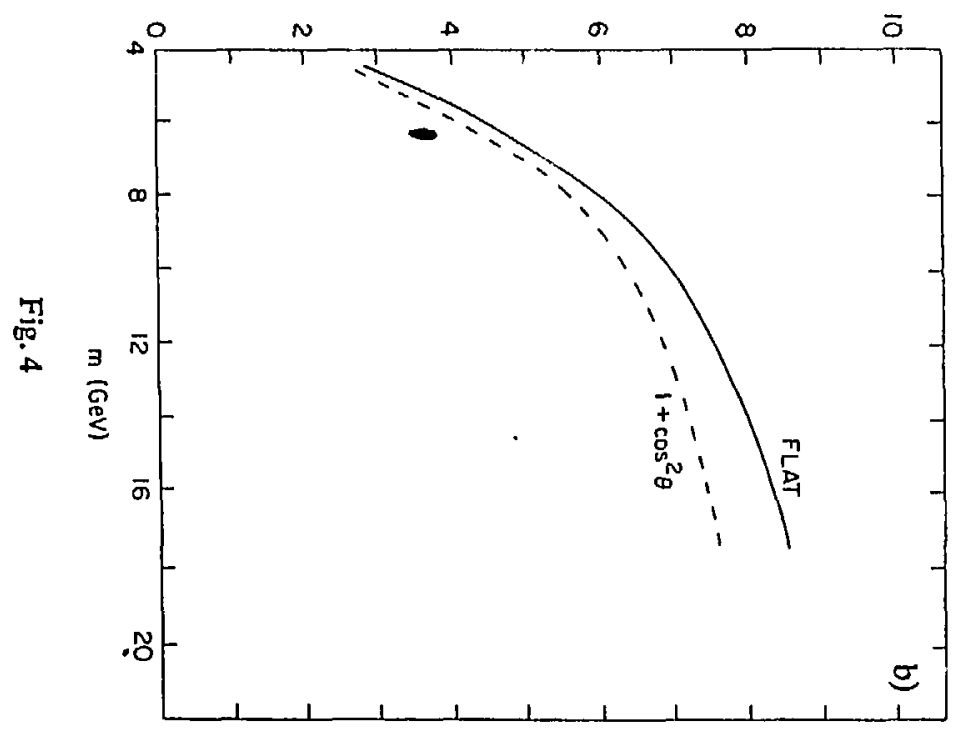

TRIGGER EFFICIENCY

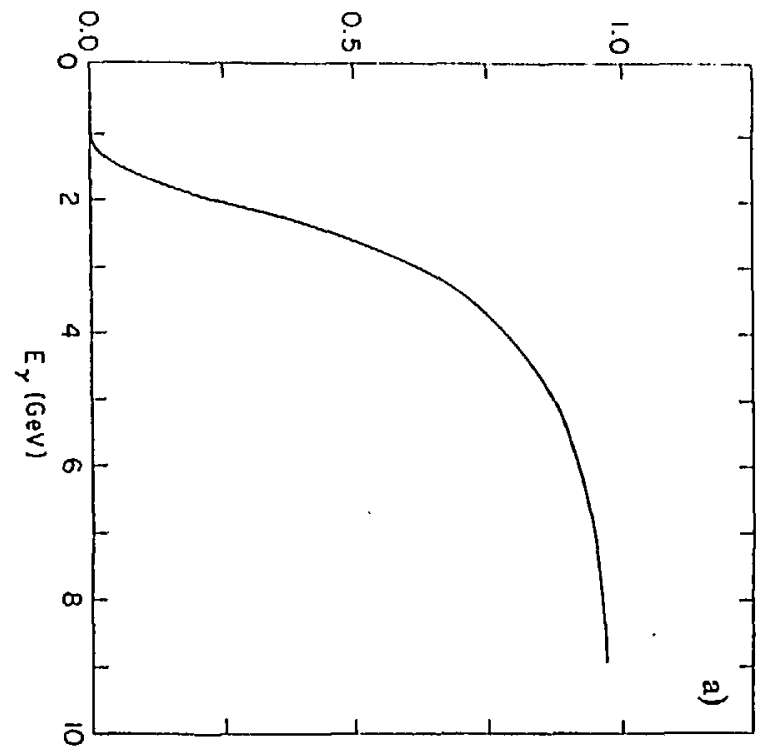




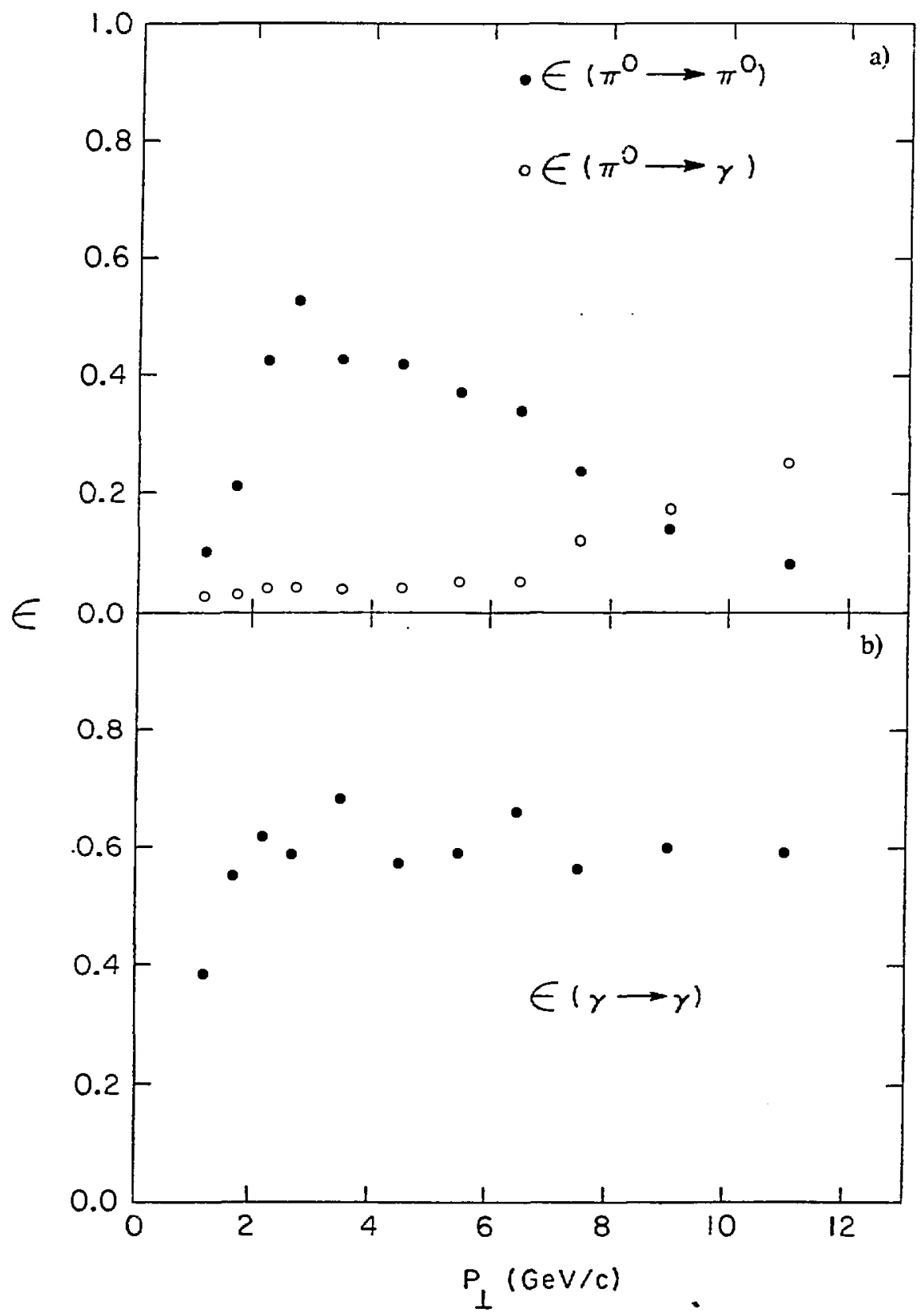

Fig. 5 


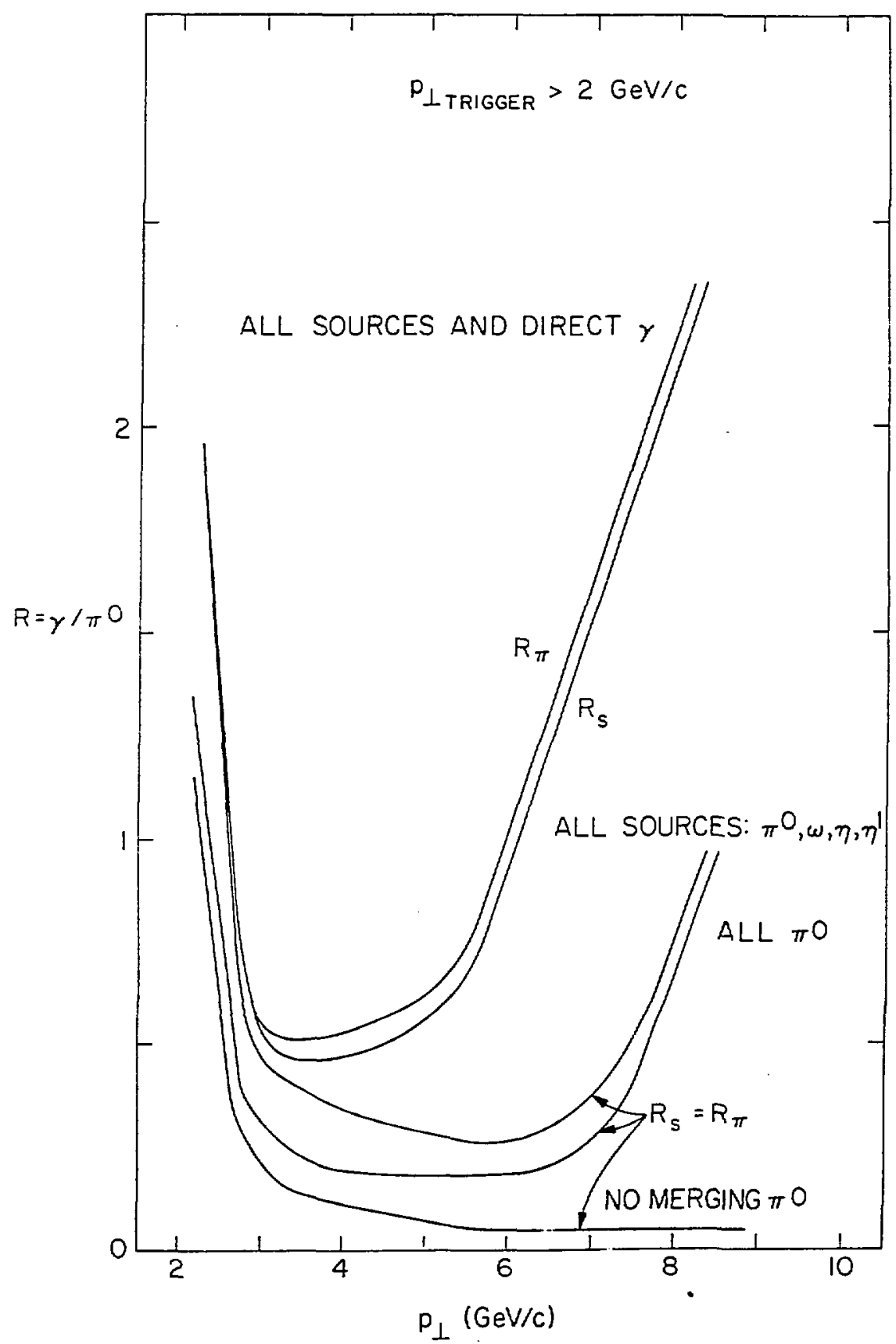

Fig. 6 


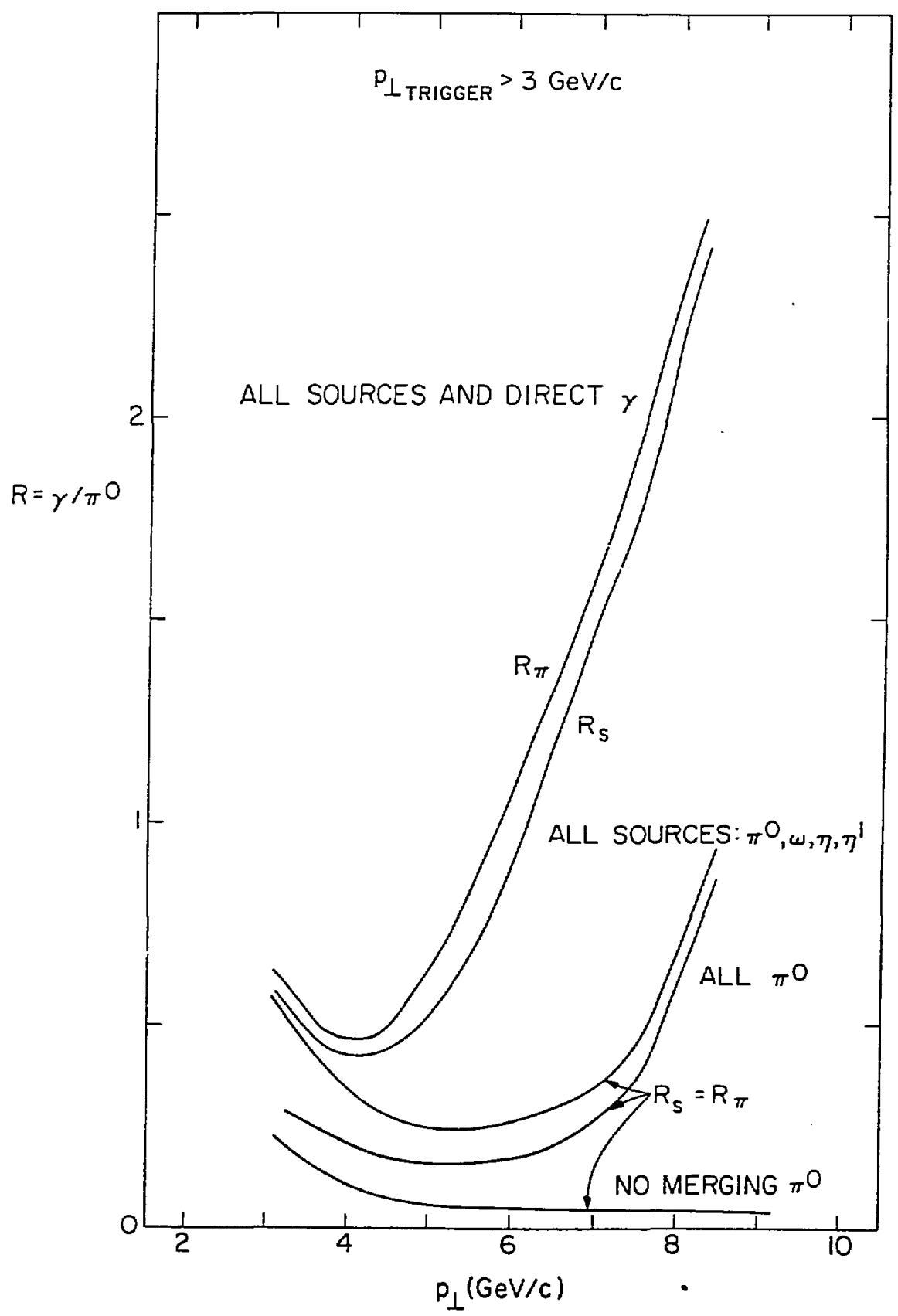

Fig. 7 


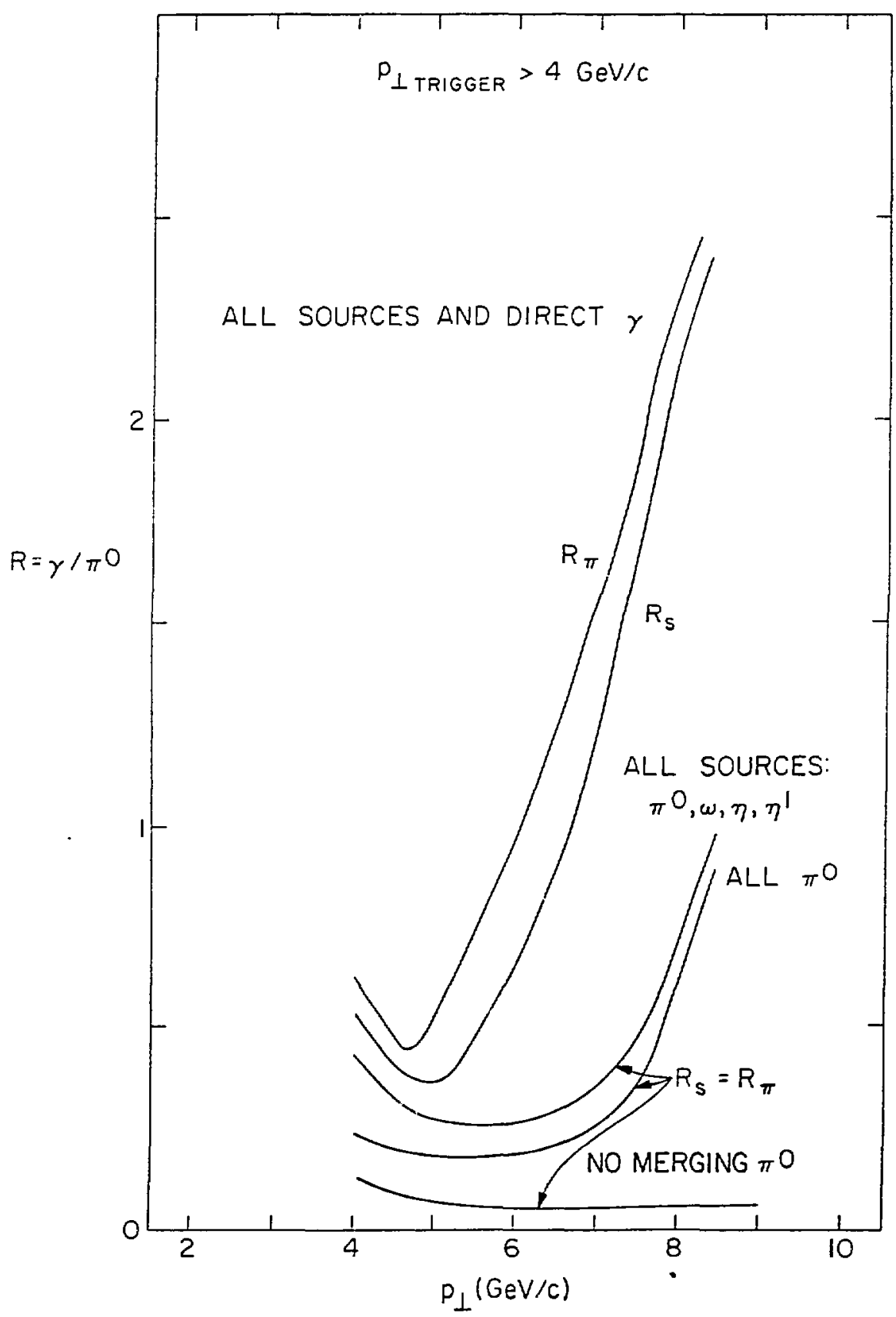

Fig. 8 


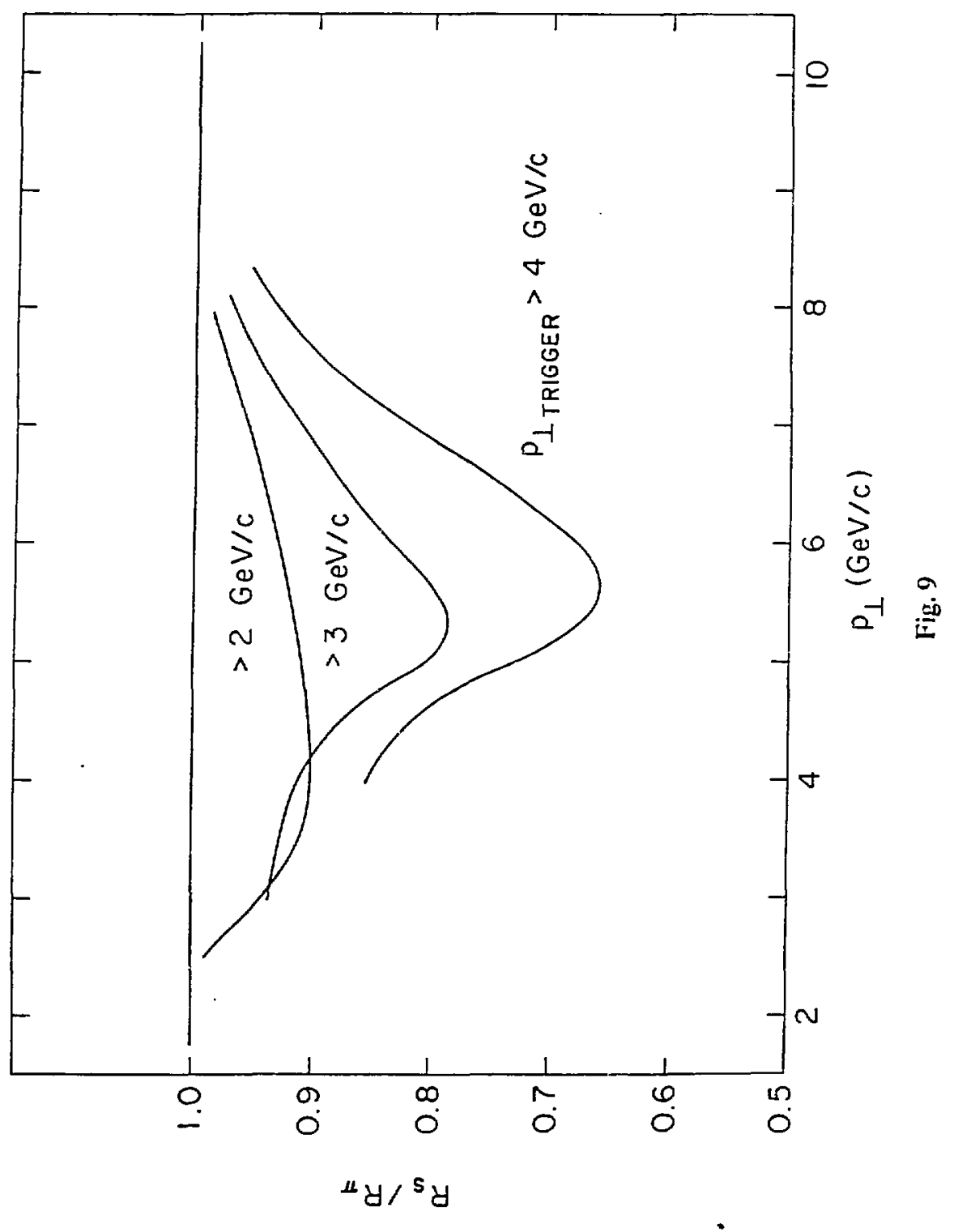



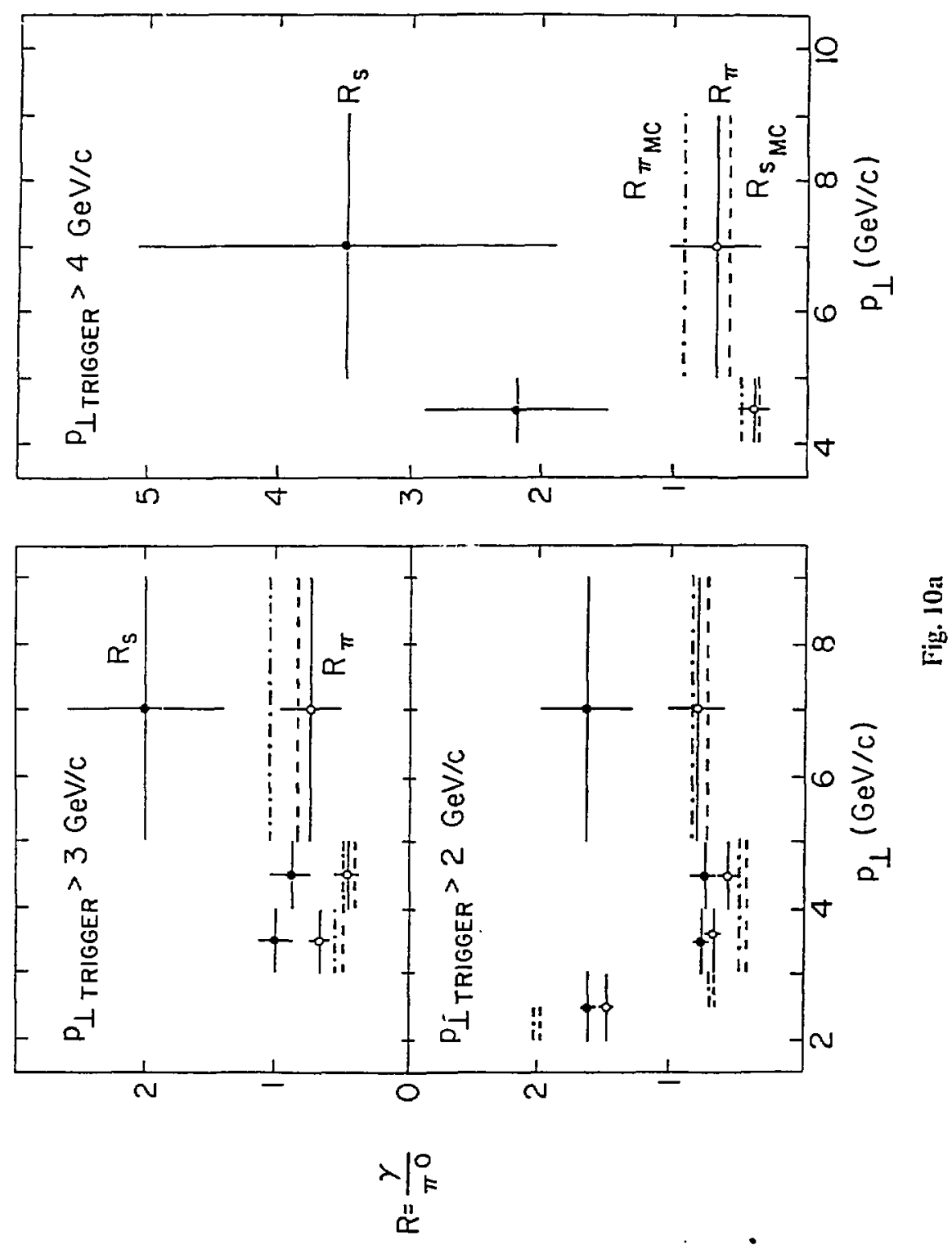

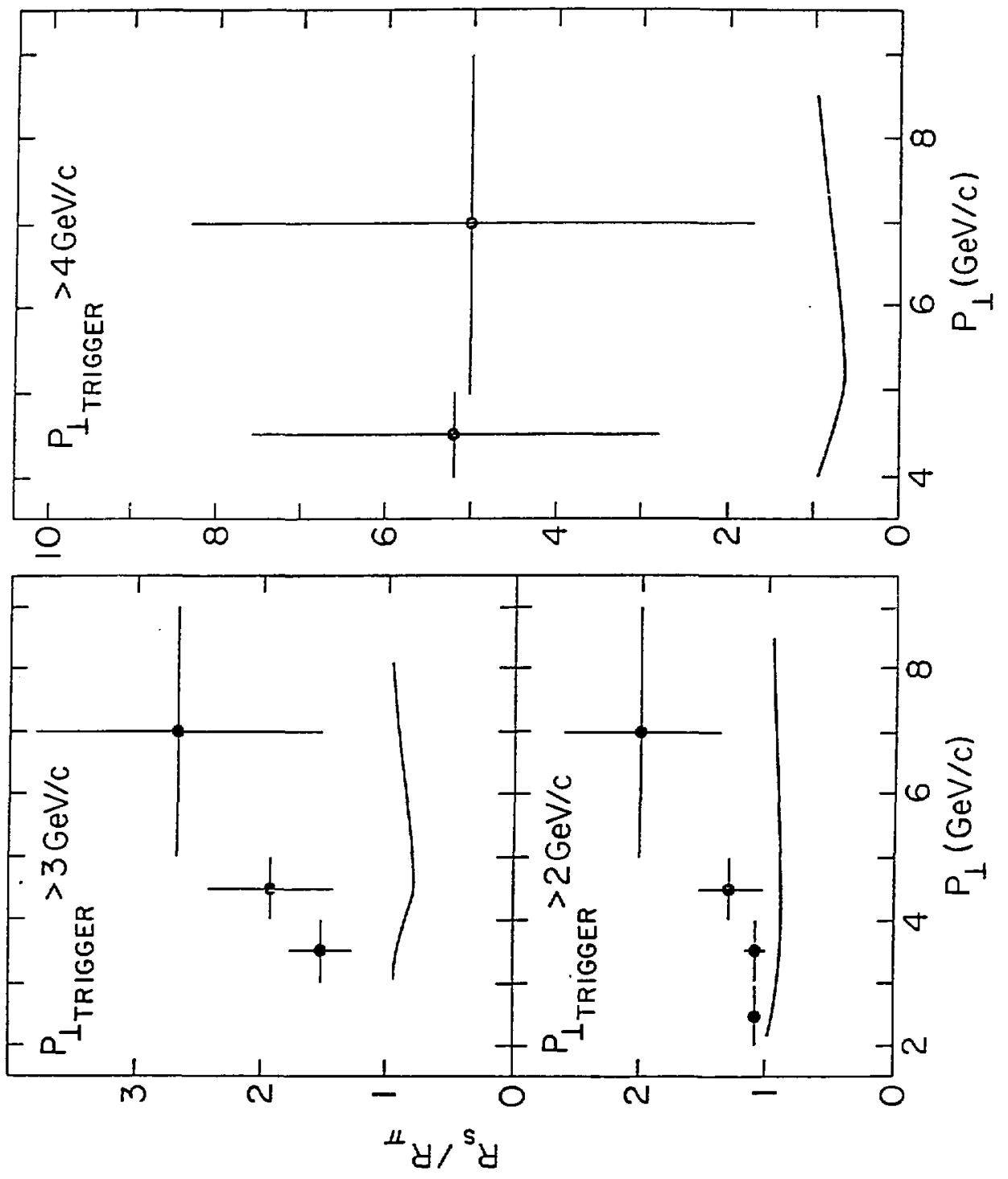

$\frac{0}{0}$ 


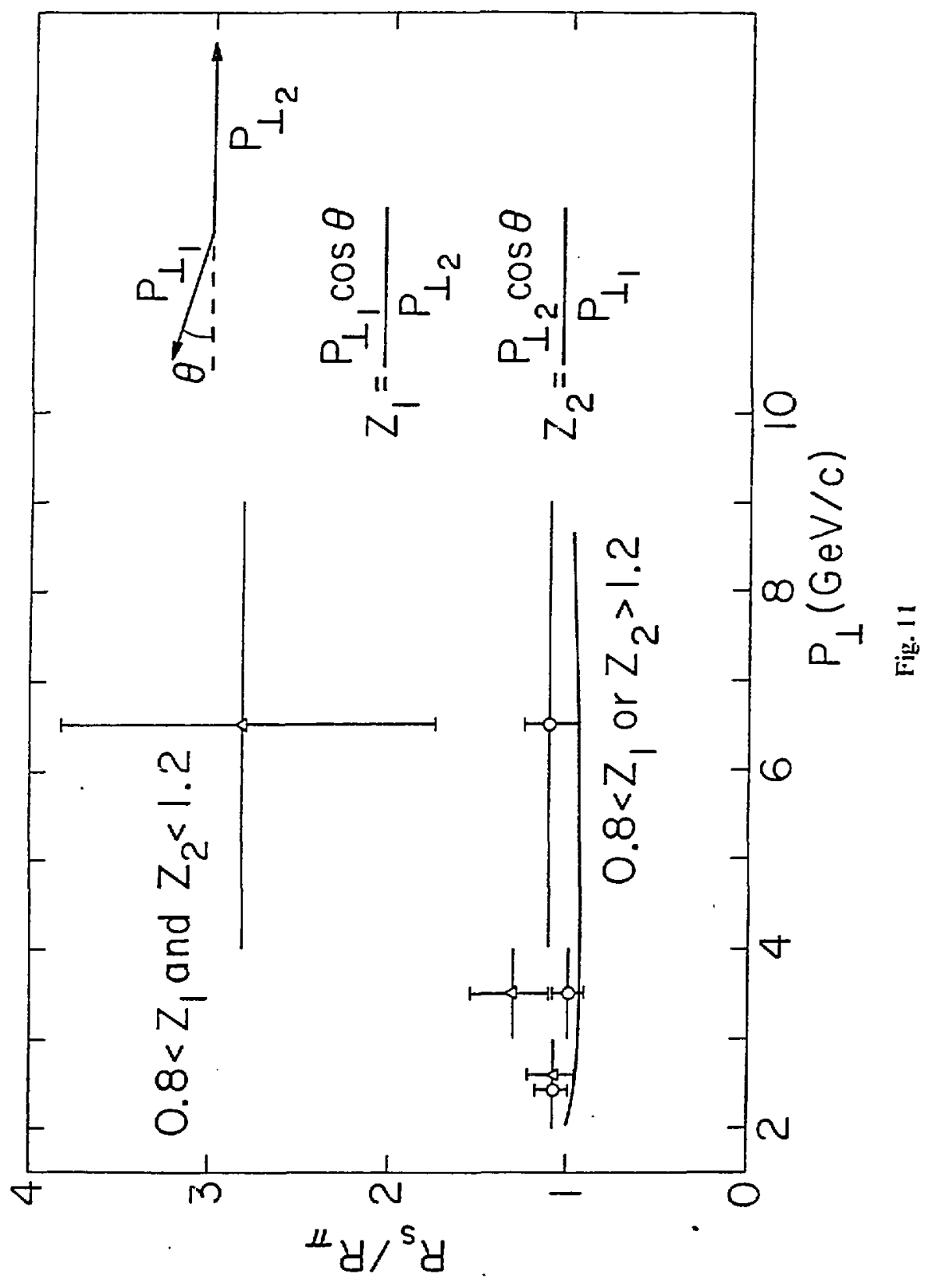




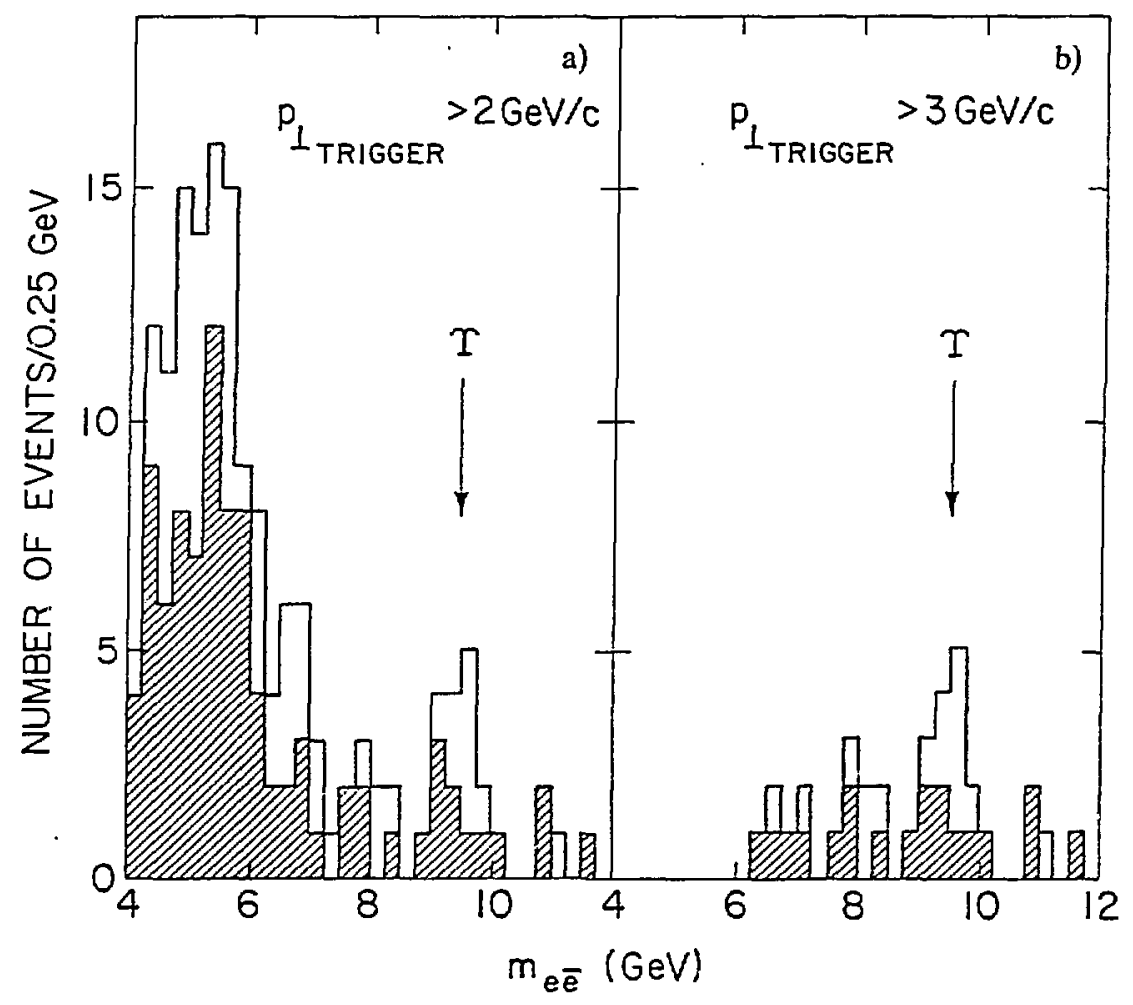

Fig. 12 